\title{
Total Basophil Count
}

National Cancer Institute

\section{Source}

National Cancer Institute. Total Basophil Count. NCI Thesaurus. Code C64470.

The determination of the absolute number of basophils in a blood sample. 